PAPER • OPEN ACCESS

Nanostars-decorated microfluidic sensors for surface enhanced Raman scattering targeting of biomolecules

To cite this article: Caterina Dallari et al 2020 J. Phys. Photonics 2024008

View the article online for updates and enhancements.
Recent citations

\begin{tabular}{l} 
- $\frac{\text { Recent progress of microfluidics in surface }}{\text { enhanced Raman spectroscopic analysis }}$ \\
\hline Ling Xia and Gongke Li \\
- Recent Advances in the Fabrication and \\
$\frac{\text { Functionalization of Flexible Optical }}{\text { Biosensors: Toward Smart Life-Sciences }}$ \\
\hline Applications \\
Bruno Miranda et al \\
- Plasmonic Nanoparticles as Optical \\
\hline Sensing Probes for the Detection of \\
Alzheimer's Disease \\
María Paz Oyarzún et al
\end{tabular}
enhanced Raman spectroscopic analysis Recent Advances in the Fabrication and Functionalization of Flexible Optical Application

- Plasmonic Nanoparticles as Optical Alzheimer's Disease María Paz Oyarzún et al 
CrossMark

OPEN ACCESS

RECEIVED

10 December 2019

REVISED

6 March 2020

ACCEPTED FOR PUBLICATION

3 April 2020

PUBLISHED

29 April 2020

Original content from

this work may be used

under the terms of the

Creative Commons

Attribution 4.0 licence.

Any further distribution

of this work must

maintain attribution to

the author(s) and the title

of the work, journal

citation and DOI.

\title{
PAPER
}

\section{Nanostars-decorated microfluidic sensors for surface enhanced Raman scattering targeting of biomolecules}

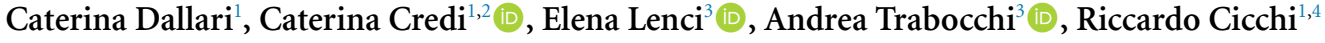 \\ and Francesco Saverio Pavone $e^{1,4,5}$ \\ 1 European Laboratory for Non-Linear Spectroscopy (LENS), University of Florence, Sesto Fiorentino (FI), Italy \\ 2 Department of Industrial Engineering (DIEF), University of Florence, Florence, Italy \\ 3 Department of Chemistry 'Ugo Schiff', University of Florence, Florence, Italy \\ 4 National Institute of Optics -National Research Council (CNR-INO), Sesto Fiorentino (FI), Italy \\ 5 Department of Physics, University of Florence, Florence, Italy \\ E-mail: credi@lens.unifi.it caterina.credi@gmail.com
}

Keywords 3D printing, surface enhanced Raman scattering, nanoparticles, microfluidics, lab-on-a-chip, nanoplasmonic sensor, bioanalytical applications

Supplementary material for this article is available online

\section{Abstract}

Novel localised surface plasmon resonance-based sensors exploitable as diagnostic devices through surface enhanced Raman scattering (SERS) represent a powerful solution for the analysis of liquid samples. In this work, we developed a rapid, versatile, low-cost and time-saving strategy combining advanced (3D-printing) and traditional manufacturing (replica molding) processes to prototype polymeric microfluidic devices, integrating all the components into a single portable platform. Microfluidics provide multiplexed capability, adequate miniaturization and robustness, handling simplicity, reliability, as well as low sample and reagents consumption, while the use of polydimethylsiloxane as supporting substrate drastically reduces the final cost. To introduce SERS capability, plasmonic features were incorporated functionalizing substrates with gold nanoparticles (NPs), engineered in terms of shape, size and surface chemistry to play with plasmonic properties as well as to guarantee reproducibility to the NPs immobilization step and consequently to the SERS effect for signal enhancing. To assess the feasibility of the measurements for molecules optical targeting, SERS-microfluidic systems were synergically coupled with a portable fiber-based set-up and Raman spectra of rhodamine $6 \mathrm{G}$ at different concentrations were acquired. To further demonstrate the potentiality of developed SERS-based substrates as point-of-care devices, Raman analysis were successfully implemented on aqueous solutions of amyloid- $\beta$ 1-42 (A $\beta)$, considered the main biomarkers for Alzheimer's disease.

\section{Introduction}

Recent researches in the field of diagnostics are more and more focused on novel methods for the molecular screening of biofluids (e.g. blood, saliva, urine, cerebrospinal fluid) as alternatives to current traditional detection bioassays, which are generally DNA-based techniques or sandwich-type immunoassays $[1,2]$. Despite being widely widespread, these tests could require long times to give response (which can range from minutes to weeks) as well as dedicated expensive equipment not accessible to every laboratory, and the accuracy could depend on the expertise of the operator [3]. In this scenario, optical detection methods based on spectroscopic techniques have been implemented in clinical diagnostic field by exploiting the non-destructive interaction between light and matter and the very rich spectroscopic information given [4]. Among all, Raman spectroscopy represents a powerful optical detection technique to be implemented because of its label-free character and non-invasive fingerprint detection capability [5]. Taking advantages of localised surface plasmons (LSPs) of metallic nanostructures and their interactions with the electromagnetic field scattered by the sample, surface enhanced Raman scattering (SERS) has been approached in order to 
enhance the Raman signal of several order of magnitude (typically $10^{6}-10^{7}$ ) and to probe low concentration analytes localized onto or near the surface of metallic nanostructures [6, 7]. The main points to be addressed when fabricating SERS-based surfaces are represented by the morphology and design of resonant metallic nanostructures and their relative distance from the target analytes. For analytical applications as well as for biosensors fabrication, the preparation of these structures has to be (i) straightforward, (ii) reproducible and (iii) cost effective to consequently (iv) guarantee a robust SERS signal [8]. To this aim, microfluidic platforms are expected to provide reproducibility of SERS analysis of liquid samples because measurements could be finely controlled on a large scale, when prone to human errors, as well as exploited when samples volumes are reduced to micron or sub-micron scale [9]. Besides this, microfluidic chips as lab-on-chip devices (LoC) can reduce the assay cost in terms of consumption of reagents, sample volumes and time. Moreover, the real possibility to perform analysis in parallel speeds up the operations while allowing the identification of multiple target analytes, avoiding cross-contamination $[10,11]$. In many ways, LoCs fulfil the requirements for point-of-care (POC) diagnostic device [12]. The promising future perspective of applying SERS-based microfluidic devices for diagnosis purposes is related to the possibility of achieving very low limit of detection ( $\mathrm{pg} \mathrm{ml}^{1}$ ) of target molecules, thanks to the preconcentration of biofluids from the extremely small quantities of dilute analytes, as the real medical cases impose [13].

The synergic combination of optical SERS sensors and microfluidic technologies is meaningful for SERS optical analysis. The flux within the microfluidic chamber enables the analyte to interact with metal nanostructures where the highest SERS enhancement is generated. Most examples of SERS-microfluidic coupling in the literature report on the injection of colloidal nanoparticles (NPs) solution and liquid samples through separated inlets of the devices placed under a microscope. Raman spectra are then collected after a period of mixing, which is crucial for the metal-analyte interaction, as well as for the difficulty to guarantee the colloidal stability upon mixing with high ionic strength bio solutions [9]. Fortuni et al described a protocol for in situ growth of Au shelled Ag-NPs on polidymethylsiloxane (PDMS), which led to great polydispersity of NPs with consequent low reproducible fabrication of SERS-substrates [14]. Moreover, most of the SERS-microfluidic POC devices described, are coupled with Raman microscopes, which are expensive bench-top bulky instruments not always compatible with measurements in a clinical scenario [15-17]. With the attempt to overcome these limitations, in the present work plasmonic microfluidic devices were rapidly prototyped and functionalized to integrate SERS sensors. The idea is to realize SERS-based microfluidic devices by combining polymeric replica molding with 3D printing stereolithography (SL) processes. Then, gold NPs is engineered to control plasmonic properties as well as NPs homogeneous immobilization on the substrates avoiding, or at least controlling, aggregation phenomena. Optically transparent SERS-microfluidic sensors were then easily interfaced with a fiber-based Raman set-up recently developed by our group, which is characterized by high portability, convenient handling and rapid acquisition times [18]. 3D printing technologies have become an attractive low-cost fabrication technology as alternative to traditional clean-room based lithography [19], since complex 3D parts of virtually any shape can be physically created or rapidly modified directly from computer-aided design files, thus enabling for product customization, process cost reduction and design versatility $[20,21]$. Then, low-cost replica molding (REM) with PDMS enables to replicate nano-sized features with the benefits of high-throughput, simple process, and the possibility to make more replicas from the same mold. PDMS is widely used because of its lower cytotoxicity, easy handling, low Young modulus and optical transparency in the UV-vis region [22-27].

Gold NPs deposition on REM-structured PDMS surfaces was achieved through silanisation of PDMS to overcome the surface chemical inertness, leading to the formation of a cross-linking self-assembled monolayer (SAM) [28]. In order to strengthen NPs-PDMS binding and to guarantee the repeatability of the immobilization step, the NPs surface chemistry was engineered by implementing a ligand exchange process to improve the colloidal stability as well as to provide new properties or functionality to the particles [29, 30]. Though the ligand exchange processes are widely described in literature for sphere-shaped NPs [31-33], few works are reported on ligand exchange for gold-nanostars, whose characteristic morphology with sharp tips causes stronger field enhancement and thus higher SERS performances respect to other NPs [17, 34, 35]. Yong Park II et al synthesized and characterized particular star-shaped particles stabilized with 11-mercaptoundecanoic acid (MUA) and methyl-PEG4-thiol MT(PEG) $)_{4}$ in order to reach a colloidal stability over time [36]. Here, the attempt was to carry nanostars ligand exchange process from native citrate to bifunctional $\alpha$-lipoic acid (LA) to exploit LA thiol groups for covalent binding with gold but also to be grafted to substrates via carboxylic acid groups. The SERS capability of AuNPs-PDMS devices coupled with portable Raman probe was first tested against rhodamine $6 \mathrm{G}$ liquid samples and then, as proof of concept, to recognize specific pathological biomarkers highly diluted within complex molecular matrices (such as biofluids). To this end, aqueous solutions of synthetic amyloid- $\beta$ 1-42 (A $\beta$ ), considered the main biomarker for Alzheimer's disease, were successfully detected. Finally, the high versatility of presented 
SERS-microfluidic devices, in terms of tailored fluidic design and tunable resonant properties, represents a great potential for the development of POCs for varied biosensing applications.

\section{Experimental section}

\subsection{Raw materials}

Gold(III) chloride trihydrate $\left(\mathrm{HAuCl}_{4} 3 \mathrm{H}_{2} \mathrm{O}\right)$; trisodium citrate dehydrate $\left(\mathrm{C}_{6} \mathrm{H}_{5} \mathrm{O}_{7} \mathrm{Na}_{3} 2 \mathrm{H}_{2} \mathrm{O}\right)$; 1-(+)-ascorbic acid (AA); silver nitrate $\left(\mathrm{AgNO}_{3}\right)$; hydrochloric acid $(\mathrm{HCl})$; nitric acid $\left(\mathrm{HNO}_{3}-70 \%\right)$; $( \pm)-\alpha$-Lipoic acid (LA); $N$-(3-Dimethylaminopropyl)-N-ethylcarbodiimide hydrochloride (EDC); $N$-Hydroxysuccinimide (NHS); (3- Aminopropyl) triethoxysilane (APTES); Rhodamine $6 \mathrm{G}$ (Rh6 G); Amyloid $\beta$ Protein Fragment 1-42; hydrogen peroxide solution $\left(30 \%\right.$ in $\left.\mathrm{H}_{2} \mathrm{O}\right)$, and all organic solvents were purchased from Merck and used without further purification. PDMS Sylgard 184 silicone elastomer was purchased from Dow Corning (Midland, MI). Vitra $430^{\circledR}$ stereolithographic photoresist was purchased from Digital Wax ${ }^{\circledR}$ Systems (DWS ${ }^{\circledR}$, Vicenza, Italy). The resin is mainly composed of urethane methacrylates with different functionalities, monofunctional monomer as reactive diluent and photoinitiators.

\subsection{SL-printing of masters and microfluidic devices fabrication}

Polymeric negative molds $(25 \mathrm{~mm} \times 35 \mathrm{~mm})$ with integrated microfluidic networks and detection chambers were designed using 'SolidWorks' software (Dassault Systèmes, France). The molds include three circular detection chambers (nominal diameter $3 \mathrm{~mm}$ ) connected to the inlet and to the outlet with microchannels of square-shaped cross section with $100 \mu \mathrm{m}$ both in width and in height. The 3D models were first processed with parametric software Nauta ${ }^{+}{ }^{\circledR}$ (DWS systems) that enables to eventually reorient the object on the working platform and then loaded to Fictor ${ }^{\circledR}$ (DWS, systems) for numerical slicing according to the user-imposed building parameters. Laser speed ranged between 250 and $5000 \mathrm{~mm} \mathrm{~s}^{-1}$, the layer thickness between $20 \mu \mathrm{m}$ and $100 \mu \mathrm{m}$. Finally, physical molds were built with a bench top XFAB $2500 \mathrm{HD}$ stereolithography apparatus (DWS systems) equipped with a monochromatic actinic laser source (Solid State Bluedge ${ }^{\circledR}$ BE-1500A/BE-1500AHR) with an emitting output power of $30 \mathrm{~mW}$ at $\lambda=405 \mathrm{~nm}$ and a $50 \mu \mathrm{m}$ laser spot diameter. At the end of the printing process, the SL-structured Vitra $430^{\circledR}$ molds were washed in ethanol to remove unreacted resin and then post-cured for $15 \mathrm{~min}$ in a dedicated ultraviolet curing unit $\left(\lambda=405 \mathrm{~nm}\right.$, Model S Ultraviolet Curing Unit, DWS $\left.{ }^{\oplus}\right)$ to accomplish the total polymer conversion. To fabricate fluidic devices, PDMS liquid precursor and its curing agent were mixed in a $10: 1 \mathrm{w} \mathrm{w}^{-1}$ and poured into the SL-printed molds. Crosslinking of the PDMS was achieved after $12 \mathrm{~h}$ at RT and $2 \mathrm{~h}$ at $70^{\circ} \mathrm{C}$. Once at RT, the PDMS replica was carefully peeled off from the molds and left again at $70{ }^{\circ} \mathrm{C}$ for $2 \mathrm{~h}$ to ensure that the PDMS is properly cured at the PDMS/air interface. Finally, sealed microfluidic devices were realized by assembling the PDMS structured replicas with a $200 \mu \mathrm{m}$ thick layer of flat PDMS after activation via air-plasma treatment. Efficient sealing is achieved through the synergic combination of hydrophobic conformal contact of the PDMS cured surfaces and the siloxane bonds formation at the interface of the two activated areas exposing hydroxyls groups $(-\mathrm{OH})[37]$.

\subsection{Nanoparticles synthesis}

Gold-nanoparticles (Au-NPs) were synthesized by the seeded-growth process described by Yuan et al [38]. The seed solution of $15 \mathrm{~nm}$-nanospheres (NSps) was prepared by adding $1.5 \mathrm{ml}$ of $30 \mathrm{mM} \mathrm{HAuCl}_{4} 3 \mathrm{H}_{2} \mathrm{O}$ (1\%) to $48.5 \mathrm{ml}$ of boiling and stirring MilliQ (stirring 7 position, $250{ }^{\circ} \mathrm{C}$ ). After $10 \mathrm{~s} 4.5 \mathrm{ml}$ of $38.8 \mathrm{mM}$ sodium citrate solution was added to the solution. Solution was stirred under heating for $15 \mathrm{~min}$, and then stirred without heating for $30 \mathrm{~min}$. For nanostars (NSts) synthesis $0.5 \mathrm{ml}$ of $30 \mathrm{mM} \mathrm{HAuCl}_{4} 3 \mathrm{H}_{2} \mathrm{O}(1 \%$ solution) was added to $19.5 \mathrm{ml}$ of MilliQ $60 \mu \mathrm{l}$ of $1 \mathrm{M} \mathrm{HCl}$ and $600 \mu \mathrm{l}$ of the NSps solution were added to the solution. Then, $600 \mu \mathrm{l}$ of $2 \mathrm{mM} \mathrm{AgNO}_{3}$ and $300 \mu \mathrm{l}$ of $0.1 \mathrm{M}$ ascorbic acid were added simultaneously. The solution was stirred for 30-60 s, while its colour turned from light red to dark grey (or blue). Immediately afterwards, NSts were centrifuged for $20 \mathrm{~min}$ at $2500 \mathrm{rpm}$ and redispersed in $100 \mu \mathrm{l}$ of distilled water. Later, $\alpha$-lipoic acid was used in a ligand-exchange reaction to replace the citrate layer with lipoic acid in both citrate-capped nanospheres (Cit-NSps) and nanostars (Cit-NSts). Au-NPs solution was diluted 1:1 with milliQ water; $100 \mu \mathrm{l} \mathrm{LA} \mathrm{(60} \mathrm{mM} \mathrm{in} 0.5 \mathrm{M} \mathrm{NaOH}$ solution) was added to the solution, which was stirred at RT for $12 \mathrm{~h}$. Lipoic acid-nanoparticles (LA-NPs) were centrifuged at $25^{\circ} \mathrm{C}, 10000 \mathrm{rpm}$ for NSps and 2500 for NSts for $20 \mathrm{~min}$ and redispersed in milliQ water.

\subsection{SERS substrates integration}

Before the sealing step, negatively-charged Cit-NPs and LA-NPs were immobilized onto PDMS surfaces through bonding formation with the amino groups $\left(\mathrm{NH}_{2}\right)$ grafted on PDMS through silanisation with aminosilane (APTES). To this end, PDMS surfaces were activated by air-plasma treatment ( $5 \mathrm{~min}$ ). Then, 
plasma-treated substrates were soaked in 1\% (v/v) APTES solutions in dried ethanol at RT for $1 \mathrm{~h}$. Hydroxylic groups $(-\mathrm{OH})$ generated on PDMS surfaces reacted with alkoxy groups from the silanes in order to covalently graft the APTES by Si-O-Si bonds formation. APTES-modified surfaces were then washed with ethanol, dried at $100{ }^{\circ} \mathrm{C}$ for $10 \mathrm{~min}$ to accomplish the silane grafting and incubated overnight with Cit- and LA-NPs solutions. Cit-NPs immobilization was achieved through electrostatic interactions of negatively-charged carboxylic acid (-COOH) groups from the citrate with positively charged $\mathrm{NH}_{2}$ from APTES-PDMS. LA-NPs were immobilized through covalent peptide bonds formation between the $-\mathrm{COOH}$ from LA with $\mathrm{NH}_{2}$ groups from APTES-PDMS. For -COOH activation, $10 \mu \mathrm{l}$ of EDC/NHS 0.4/0.1 M aqueous solution were added every $\mathrm{ml}$ of LA-NPs solution diluted 1:1 in milliQ water and stirred for $30 \mathrm{~min}$ at RT. Immediately after, LA-NPs solution with $\mathrm{COOH}$-activated groups were poured on the APTED-PDMS samples and incubated overnight. Weakly bound NPs were removed by washing thrice with MilliQ water and drying with nitrogen. From here on substrates will be named for brevity as follow: PDMS decorated with citrate-capped nanospheres: Cit-NSps-PDMS; PDMS decorated with citrate-capped nanostars: Cit-NSts-PDMS; PDMS decorated with lipoic acid-capped nanospheres: LA-NSps-PDMS; PDMS decorated with lipoic acid-capped nanostars: LA-NSts-PDMS.

\subsection{Characterization techniques}

The absorbance spectra of gold NPs colloidal solutions and the plasmonic properties of PDMS substrates modified with NPs were acquired in the range from $400 \mathrm{~nm}$ to $850 \mathrm{~nm}$ with a UV-vis spectrophotometer (Lambda 950 instrument, Perkin Elmer). UV WinLab Software was used to acquire spectra and data were processed with Origin. UV-vis spectra for NPs-PDMS substrates were acquired by vertically fixing the samples to the sidewall of the standard cuvette filled with water. The hydrodynamic dimensions and distributions were characterized by Dynamic Light Scattering (DLS) analysis performed with a Malvern Zetasizer Nano series ZS90. Data were processed with Origin software. The NPs morphology in terms of size and shape was characterized by transmission electron microscope (TEM, CM 12 PHILIPS). Proton nuclear magnetic resonance $\left({ }^{1} \mathrm{H}-\mathrm{NMR}\right)$ was conducted on a Varian Inova $400 \mathrm{MHz}$. For the analysis, lyophilized NPs $\left(3 \mathrm{mg}\right.$ at $\left.\mathrm{T} 22{ }^{\circ} \mathrm{C}\right)$ were dissolved in deuterated water $\left(\mathrm{D}_{2} \mathrm{O}, 0.6 \mathrm{ml}\right)$. Standard ${ }^{1} \mathrm{H}$ experiment was performed using a minimum recycle delay of $5 \mathrm{~s}$.

\subsection{Raman-SERS measurements}

The Raman set-up to be coupled with the devices was developed by our group and detailed description is reported elsewhere in the literature [18]. Briefly, we exploited a laser diode emitting at $785 \mathrm{~nm}$ with $0.2 \mathrm{~nm}$ spectral bandwidth (LD-785 nm-FC-785-350-MM2-PC-1-0-RM, RGB lase, Fremont, CA, US) coupled to the Raman probe (EMVision LCC, Laxahatchee, FL, US) by a FC/PC connector. The probe consists of a bifurcated bundle with 25 optical fibers ( $100 \mu \mathrm{m}$ core diameter, low -OH content, $0.22 \mathrm{NA}$ ): 24 for collecting the signal arranged in a honeycomb fashion around the central one used for light delivering. A

$785 \mathrm{~nm}$-narrow band pass filter and a ring-shaped long pass filter are placed at the distal end of the probe in order to clean the laser line by blocking the unwanted fluorescence and spurious Raman generated inside the excitation fibre. The bundle of fibers and the filters are held together by a metallic jacket with an outer diameter of $2.1 \mathrm{~mm}$. A MTP connector is used to connect the collecting fibers to the detection system that is composed of a monochromator (microHR HORIBA Scientific Edicon, NJ, US) equipped with a 600 lines $\mathrm{mm}^{-1}$ grating, a filter wheel hosting LP02-785RE-25 (Semrock, Rochester, NY, US) for Raman detection, a notch filter at $785 \mathrm{~nm}$ (NF03-785 $\times 10^{-25}$, Semrock, Rochester, NY, US) to prevent the detection of Rayleigh back-scattered photons and a $-60^{\circ} \mathrm{C}$ cooled CCD array camera (Syncerity HORIBA Scientific, Edicon, NJ, US) connected to the output port. For Raman spectra acquisition, liquid samples were injected with a syringe into the microfluidic channels through the dedicated inlet and the distal end of the spectroscopic probe was vertically aligned leaning respect to the microfluidic devices in correspondence of the SERS-active detection chambers. SERS spectra were acquired in the wavenumber range of $900-1800 \mathrm{~cm}^{-1}$. To avoid spurious signals, the bottom layer of the PDMS devices were coupled with calcium fluoride Raman slides $\left(\mathrm{CaF}_{2}\right.$, Crystan). The laser power at the solution was about $150 \mathrm{~mW}$, the acquisition time was $5 \mathrm{~s}$ and the measurement were repeated ten times for spectral averaging. In order to extract the Raman signal of interest, fluorescence and background signals were subtracted from the acquired raw spectra through Vancouver Raman Algorithm, a dedicated software for automatic autofluorescence back-ground subtraction for Raman spectroscopy [39]. Data were further analysed with Origin software.

\section{Results and discussion}

Hybrid organic-inorganic microfluidic devices for the optical analyses of liquid biopsies are presented. To this end, a strategy combining advanced and traditional manufacturing processes with gold NPs engineering 


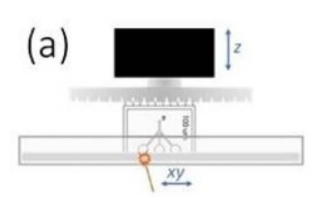

(b)
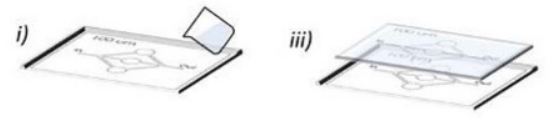

ii)

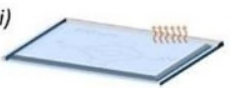

vi)
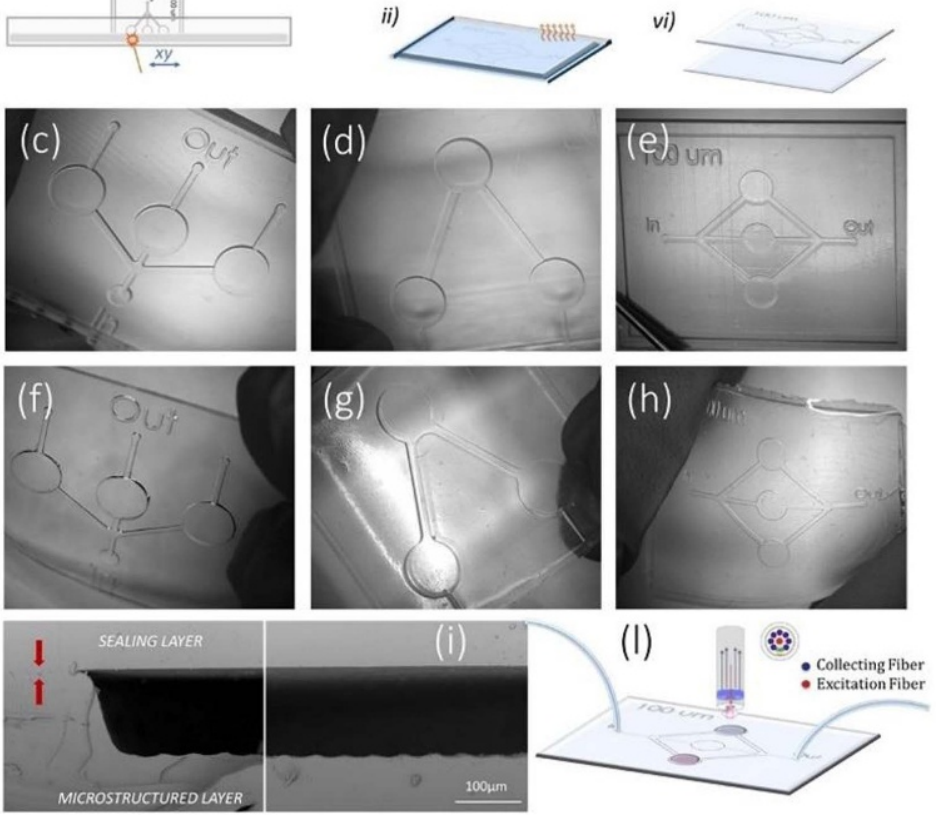

Figure 1.: (a) SL-fabrication process of plastic mold; (b) scheme representing REM-fabrication of monolithic PDMS devices: (i) pouring the PDMS prepolymer mixture in the SL-mold, (ii) thermal curing of PDMS, (iii) peeling off the mold and (iv) sealing with a flat layer of cured PDMS. MO images of SL-printed molds with varied designs (c), (d) and (e) and corresponding PDMS elastomeric replica (f), (g) and (h). SEM images of the cross section of the detection chamber (i). Scheme depicting the final device coupled with the Raman fiber probe (l).

in terms of shape, size and surface chemistry has been optimized to rapidly fabricate SERS-active sensors in a low-cost and time-saving way while guaranteeing high design flexibility, substrate stability and reproducibility. First, PDMS microfluidic devices were obtained by REM of plastic molds fabricated via stereolithography (SL) within $30 \mathrm{~min}$ (Scheme in figures 1(a) and (b)). As a matter of fact, devices rearranged with channels and detection chambers characterized by customized geometrical features can be rapidly prototyped. As shown in figures 1(c), (d) and (e), the general design presented includes three circular-shaped detection chambers connected by a simple network of microchannels with rectangular-shaped cross section $(200 \mu \mathrm{m}$ in width and $100 \mu \mathrm{m}$ in height). The huge dimension of the chambers $(\phi=3 \mathrm{~mm})$ respect to the excitation laser spot and their relative spacing can be exploited to perform Raman-SERS measurements at different conditions (one per chamber) avoiding cross contamination and in a very fast process by moving/shifting horizontally the probe. On the other hand, being the laser irradiance proportional to the inverse square of the distance, in order to increase the efficiency of the Raman signal delivering and collection, the nominal z-depth of the chambers was $100 \mu \mathrm{m}$, corresponding to the accuracy of the SL-printer exploited.

Finally, the overall dimensions of the devices $(25 \mathrm{~mm} \times 35 \mathrm{~mm})$ were considered to optimize the integration of the microfluidic functional components while guaranteeing easy handling, allowing for routine analysis. Indeed, 3D printing technologies enable for design engineering as a function of the final application of the device overcoming expensive and time-consuming silicon-based machining processes which also limit customization and design flexibility. PDMS microstructured devices were obtained by REM the SL-printed molds (figures $1(\mathrm{f}),(\mathrm{g})$, and $(\mathrm{h})$ ). Great advantage is also represented by the real feasibility to create many PDMS replicas (more than 20 times) before the quality of the molds are degraded, thus clearly highlighting the standardization of this method. PDMS was selected not only for its evident high-fidelity REM capability, but mainly for its optically transparency in the NIR region, which should permit to perform Raman analysis in the NIR region working in backward configuration. Monolithic PDMS devices were obtained by covalent siloxane bonds formation ( $\mathrm{Si}-\mathrm{O}-\mathrm{Si}$ ) upon bringing together air plasma-treated flat and microstructured PDMS layers. The SEM images in figure 1(i) show the cross section of a detection chamber after the sealing step with the two PDMS layers jointed without any interfaces. The wavy textures observed for the bottom surfaces of the microstructured layer is typical of the laser-scanning SL- construction method but it did not alter the bonding step, as no leakage phenomena occurred. Furthermore, despite the huge area 
covered by the chambers, no clogging or warping phenomena were observed for the flat layers covering the chambers. To realize SERS substrates within the detection chambers we started from preliminary results recently obtained by our group [28] and we further optimized the process of immobilization of gold NPs onto PDMS. Aiming at improving SERS substrates efficiency and standardization, in the present study the NPs surface chemistry was engineered to strengthen NPs colloidal stability as well as NPs-PDMS binding. To this end, a ligand exchange process from native citrate (Cit) protecting agent to lipoic acid (LA) was successfully implemented for gold nanospheres (NSps) and for the first time on gold nanostars (NSts). LA has a disulphide bridge (S-S) that is reduced to thiols groups for anchoring to the NPs surfaces because of the strong affinity of sulfur to gold. Thus, switching from electrostatic (Cit-Au) to covalent (LA-Au), NPs-ligand interactions is expected to overcome ligands molecules desorption caused by changes in $\mathrm{pH}$, ionic strength or substrate incubation (e.g. with PDMS) that lead to NPs not reversible aggregation with blue shift in UV-visible absorption. Furthermore, the carboxylic acid end-chain group from LA is exploitable for further functionalization or anchoring, as deeply described later.

Extinction spectra reported in figures $2(a)$ and (b) demonstrated that we successfully accomplished covalent LA anchoring for both NSps and NSts, whose complex morphology with high aspect ratio sharp tips could affect the homogeneity of the exchange process. A little red-shift is observed for LA-NPs spectra respect to the Cit-NPs (both spheres and stars). This could be ascribed to the different chemical environment and bond characteristic of the two ligands directly affecting the extinction coefficient and therefore the maximum absorbance wavelength. Lipoic acid chemisorbs directly to the surface of gold NPs, causing an increase in local refractive index [40]. $\Delta \lambda$ calculated from the UV-visible spectra reported in figures 2 (a) and (b) are equal to $4 \mathrm{~nm}$ for NSps and to $10 \mathrm{~nm}$ for NSts: this is probably due to a higher sensitivity to refractive index changes of the branches of NSts respect to spheres-core plasmons [17]. On the other hand, from TEM images (figureS1 in supporting information) no significant modifications were observed in NPs core size upon exchange of stabilizing agent. The size of Cit-NSps was $16.5 \pm 0.2 \mathrm{~nm}$ and resulted comparable to LA-NSps whose DLS- diameter was $22.8 \pm 0.9 \mathrm{~nm}$. The same trend of slightly increasing in hydrodynamic dimension upon ligand exchange was measured for NSts with values from $90.8 \pm 0.6 \mathrm{~nm}$ to $102.4 \pm 0.4 \mathrm{~nm}$ for Cit-NSts and LA-NSts, respectively (figures $2(\mathrm{c})$ and $(\mathrm{d})$ ). Furthermore, zeta potential $(\zeta)$ measurements confirmed that citrate to lipoic acid ligand exchange was successfully obtained with $\zeta$ increasing from $-22.7 \mathrm{mV}$ for citrate to $-28.8 \mathrm{mV}$ for lipoic acid. The $\zeta$ measured for LA-NSts can be ascribed to a self-assembled monolayer completely covering the metal surface with few molecules orienting deprotonated carboxyl groups toward metal surface due to electrostatic interfacial energy [40]. ${ }^{1} \mathrm{H}-\mathrm{NMR}$ spectra of LA-NSts further confirmed that successful ligands exchange was achieved. As shown in figure 2(e), the peaks related to LA appear significantly broadened, shifted or sometimes even completely disappeared, due to the distribution of the chemical environment [41]. The differences of chemical shift between free and surface-bound ligand are related to the distance of the analysed proton from the NPs surface [40]. For LA-NSts, the ${ }^{1} \mathrm{H}$ resonances of the protons attached to $\mathrm{C}_{1}$ completely disappeared, while ${ }^{1} \mathrm{H}$ peak of protons attached to $\mathrm{C}_{2}$ was shifted and seriously broadened. The methylene protons of the carbon of the aliphatic chain of lipoic acid are around $0.1 \mathrm{ppm}$ or even no shifted respect to lipoic acid free molecule, thus protons on carbons 1 and 2, which are closer to the gold particle surface, experienced a higher shift of about $0.2 \mathrm{ppm}$. Narrow and well-defined peaks related to free lipoic acid are present in figure 2(e), which states for unbound LA molecules still present in the colloidal dispersion. Finally, the real possibility to engineer the NSts surface chemistry despite the complex morphology is of great interest for their intrinsic resonant properties, as well as their noteworthy enhancing performances. Once LA-capping was confirmed, a bottom-up strategy involving the grafting of intermediate linker was implemented to selectively decor the PDMS detection chambers with NPs. PDMS chemical inertness was overcome by grafting $\mathrm{NH}_{2}$ groups through silanisation with APTES. To spatially confine the NPs and to avoid undesired aspecific absorption, the silanisation process was selectively implemented for the PDMS chambers. Then, the $-\mathrm{COOH}$ end groups from the bifunctional LA were exploited for LA-NPs covalent immobilization through peptide bonds formation with $\mathrm{NH}_{2}$ from APTES-PDMS. Therefore, strengthened anchoring should be guaranteed for LA-NPs-PDMS respect to Cit-NPs-PDMS weakly bound through electrostatic interactions (figure 3(a)). Even if optical densities (O.D.) of both NSps-substrates shown in figure 3(b) are comparable, the LA-NSps-PDMS absorbance contribution results to be 2-folds higher with respect to Cit-NSps considering its higher baseline which states for a scattering cross section increased due to aggregation. Concerning NSts, LA-NSts-PDMS substrates (figure 3(c)) clearly present higher O.D., corresponding to higher density of deposited NPs even after washing steps. Moreover, LA-NPs-PDMS samples retained the typical shape of the colloidal NPs solution used respect to Cit-NPs-PDMS samples, which is crucial for SERS. The wavelength shift and the broadening of the curves observed for Cit-NPs-PDMS are evidence of aggregation phenomena and particles clustering with consequent alteration of the plasmonic properties. During the incubation process on the substrate, citrate could desorb totally or partially thus decreasing the inter-particles distance randomly. On 


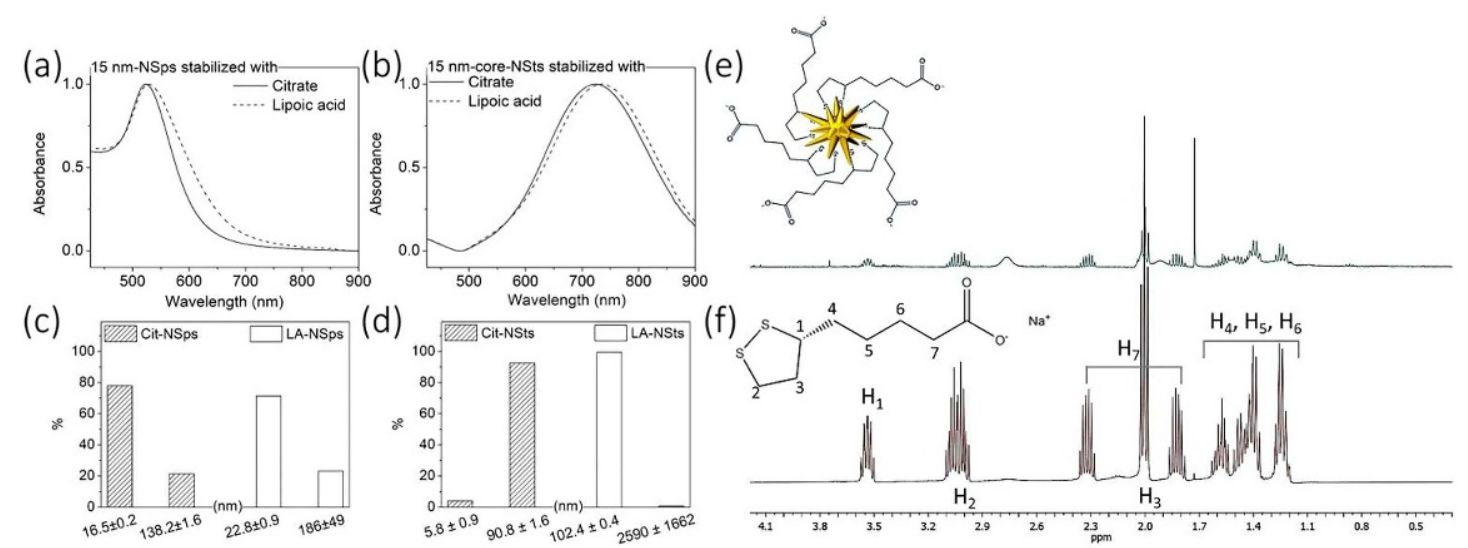

Figure 2. Extinction spectra of gold nanospheres (a) and nanostars (b) colloidal solutions before and after the ligand exchange process. Size distribution for nanospheres (c) and nanostars (d). ${ }^{1}$ H-NMR of lipoic acid-nanostars (e) and lipoic acid free molecule (f).

(a) Electrostatic interactions

(b)
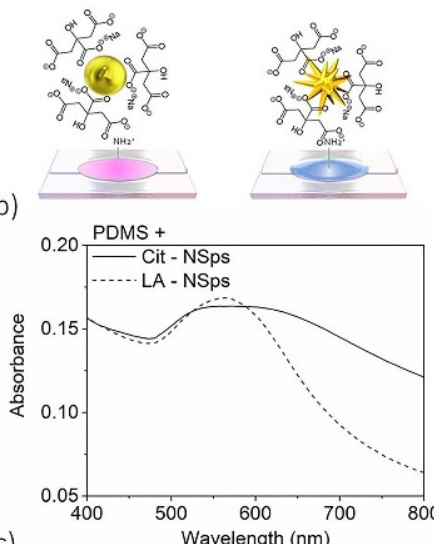

(c)

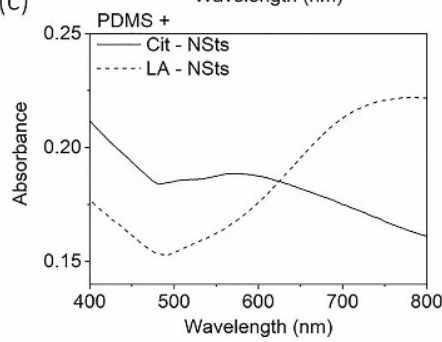

(d)

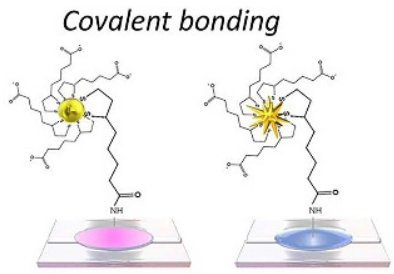

(e)
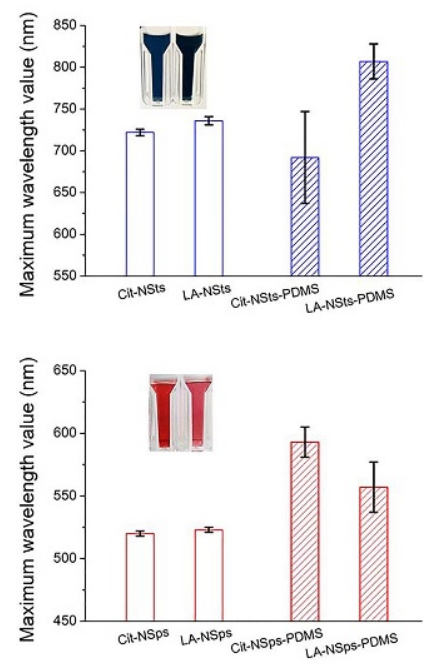

Figure 3. (a) Scheme of the chemical functionalization of PDMS substrates with Cit- and LA-capped particles. UV-vis extinction spectra acquired on APTES-PDMS decorated with nanospheres (b) and nanostars (c) with different stabilizing agent. Mean plasmonic resonance values measured for Cit- and LA-NSps in solution and deposited on PDMS (d) and Cit- and LA-NSts in solution and deposited on PDMS (e) for nanostars.

the other hand, being LA covalently anchored to the gold surfaces, particles retain at least LA chain length spacing upon aggregation.

Then, to further verify the real possibility to ensure high repeatability of SERS-substrate fabrication process, the plasmonic properties of Cit-NPs-PDMS and LA-NPs-PDMS samples were compared with those of colloidal solutions in terms of the mean absorbance values and relative standard deviation. From the histograms reported in figures 3(d) and (e), it is evident that Cit- and LA-NPs-PDMS samples (both spheres and stars) are characterized by different values respect to the colloidal solutions. This is obvious considering that we moved from colloidal systems to immobilized NPs. The trend of red-shifting (70 nm for Cit-NSps, $34 \mathrm{~nm}$ for LA-NSps and $71 \mathrm{~nm}$ for LA-NSts) respect to the corresponding colloidal solutions is due to a decrease of the inter-particles distances when aggregating, which led to SPR at higher wavelengths. It is important to highlight the higher deviation of the Cit-NPs-PDMS samples confirming the weakness of citrate stabilization. Cit-NSts-PDMS experienced a blue shift for a progressive disappearance of tips due to a 

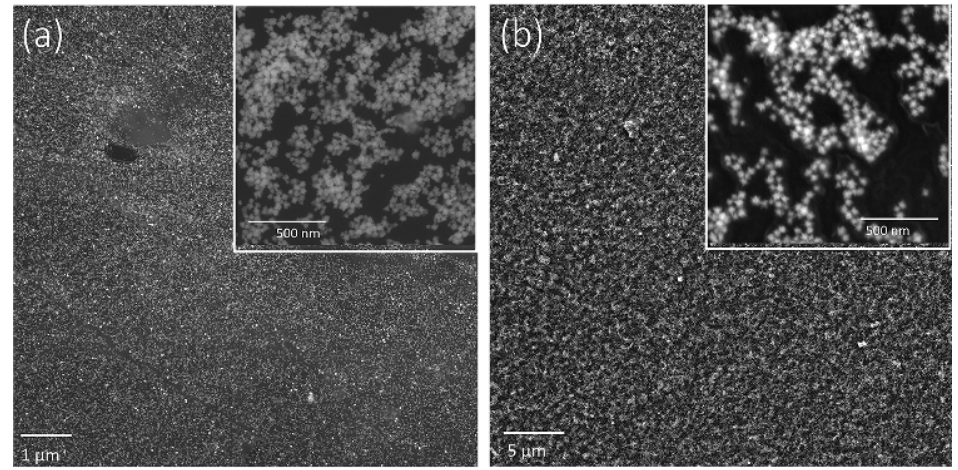

Figure 4. SEM images of closely packed multilayer structure of particles (a) Cit-NSts and (b) LA-NSts immobilized onto PDMS silanized-surface. The insets show higher magnification images (120x).

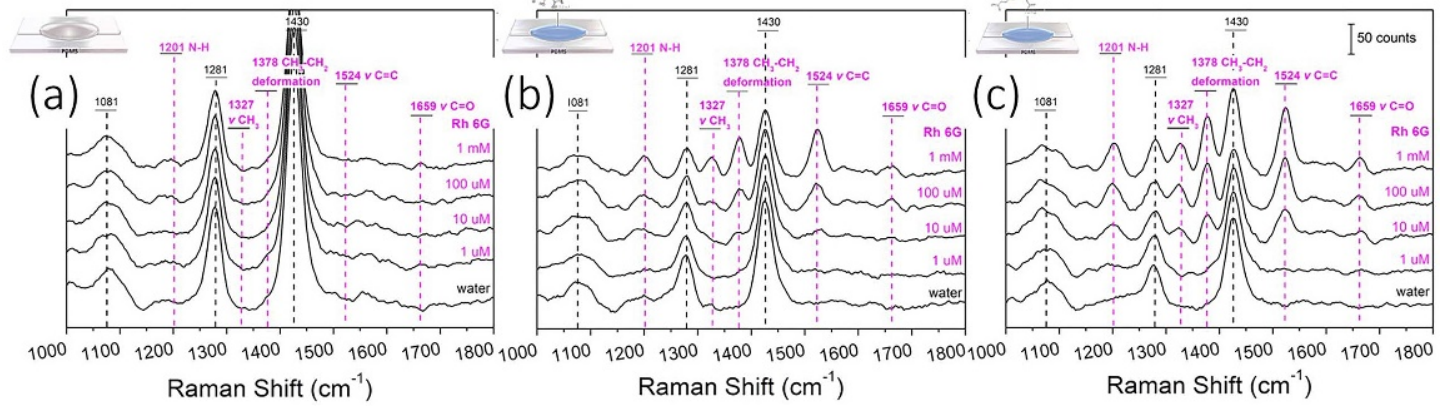

Figure 5. Raman-SERS spectra of Rhodamine $6 \mathrm{G}$ aqueous solution at concentration ranging from $1 \mathrm{mM}$ to $1 \mu \mathrm{M}$, acquired aligning the distal end of the Raman fiber with: bare PDMS microfluidic devices (a), Cit-capped (b) and LA-capped (c) nanostars. Wavenumbers were assigned to relative peaks. Both types of particles are SERS-active, enhancing Raman peaks respect to bare PDMS.

reorganization of its outer gold atoms characterized by high-electrostatic potential energy [42]. As previously stated, LA-NSts-PDMS maintain the minimum LA-chain distance with collapse tips not collapsing into each other, and thus controlling assembling and presence of hot-spot. Consequently, moving from colloidal solutions to substrates, LA-samples retain the resonant properties on average, expressed by low deviation values. No significant differences were observed from SEM images of Cit-NSts-PDMS and LA-NSts-PDMS reported (figure 4), both showing uniform layer of deposited particles.

\subsection{Raman-SERS measurements}

To demonstrate the potentiality of developed SERS-based devices as POCs, NPs decorated PDMS microfluidic detection chambers were aligned with the distal end of the Raman fiber probe, which simultaneously emits the excitation radiation and collects the inelastic scattered signal. Rhodamine $6 \mathrm{G}$ (Rh6 G) aqueous solutions at different concentration ( $1 \mathrm{mM}, 100 \mu \mathrm{M}, 10 \mu \mathrm{M}, 1 \mu \mathrm{M})$ were fluxed through the microchannels and Raman spectra were immediately acquired within less than a minute without pre-incubation of the SERS substrates. As shown in figure 5, narrow peaks characteristic of both Rh6 G and PDMS were found in the spectra acquired for all the different substrates [43].

Being the devices constituted by bulky monolithic PDMS system, the Raman peaks $1081 \mathrm{~cm}^{-1}$ and $\mathrm{CH}_{3}$ symmetric and asymmetric bending at $1284 \mathrm{~cm}^{-1}$ and $1432 \mathrm{~cm}^{-1}$ (black dot line in figures 5 (a)-(c)) can be assigned to the polymer. This was further confirmed by the fact that these peaks did not vary when increasing Rh6 G concentration. Conversely, Rh6 G Raman peaks at $1201 \mathrm{~cm}^{-1}$ (aromatic ring N-H), $1327 \mathrm{~cm}^{-1}\left(\mathrm{CH}_{3}\right.$ umbrella mode), $1378 \mathrm{~cm}^{-1}\left(\mathrm{CH}_{3}-\mathrm{CH}_{2}\right.$ deformation), $1524 \mathrm{~cm}^{-1}$ (aromatic $\mathrm{C}=\mathrm{C}$ stretching) and $1659 \mathrm{~cm}^{-1}$ ( $\mathrm{C}=\mathrm{O}$ stretching) were observed to appear and to progressively vary in intensity when increasing from water (no Rh6 G) to $1 \mathrm{mM}$ Rh6 G aqueous solution. Graphs reported for bare PDMS chambers (figure 5(a)) are mainly characterized by high PDMS peaks with Rh6 G profile slightly visible just for the $1 \mathrm{mM}$ highest concentration. Instead, SERS effect was observed for both Cit-NSts-PDMS and LA-NSts-PDMS devices (figures 5(b) and (c)), with Rh6 G peaks enhanced at all concentrations, even one order of magnitude lower than examples reported in literature [16]. As expected, a relation between SERS 

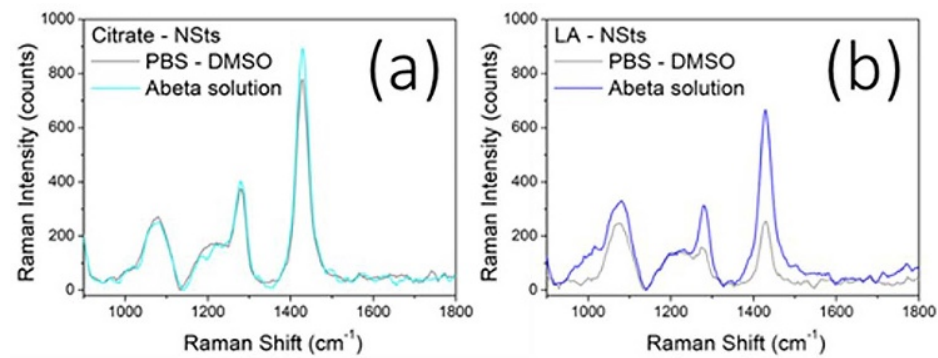

$A \beta$ aqueous solution

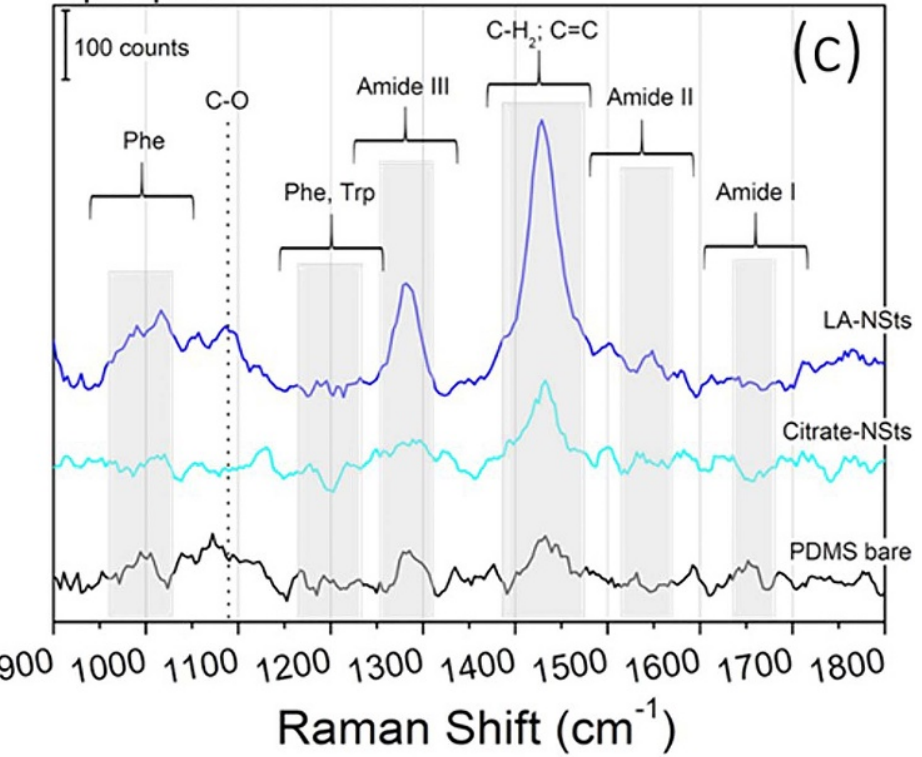

Figure 6. Raman spectra of a $\beta$-42-peptide against buffer solution for Cit-NSts substrates (a) and LA-NSts-PDMS (b). (c) Comparison of the background-subtracted Raman 'fingerprint' spectral region of a $\beta$-42-peptide between bare PDMS, Cit-NSts, LA-NSts substrates. Spectra have been shifted vertically for clarity of presentation and wavenumbers are listed above each peak. Abbreviations: Phe-phenylalanine; Trp—tryptophan.

enhancement with the resonant properties of NPs-decorated chambers emerged. More into detail, the higher enhancement was obtained with NPs-PDMS which has the higher absorbance value at $785 \mathrm{~nm}$, thus in resonance with the excitation wavelength of the setup. The results demonstrated that the developed SERS-based devices, synergically coupling optics and fluidics, can be exploited for Raman analyses of liquid matrices to detect diluted analytes. To attest the repeatability of SERS measurements, a crucial role in POCs devices, several spectra of Rhodamine $6 \mathrm{G}$ at different concentrations have been acquired by testing Cit-NSts and LA-NSts substrates fabricated from different batches. The repeatability of SERS measurements was investigated by monitoring the variation in the intensity of two peaks ascribable to rhodamine $\left(1524 \mathrm{~cm}^{-1}\right.$ and $1201 \mathrm{~cm}^{-1}$ ). As shown in figure S5, peak intensity increases by increasing in Rh6 G concentration for both LA-NPs and Cit-NPs substrates. However, the deviation standard values obtained for LA-NPs decorated samples are lower respect to Cit-NPs samples at each concentration, thus attesting the higher reproducibility of SERS measurements and further confirming the improved performances of lipoic acid-NPs, already demonstrated through O.D measurements.

Finally, to demonstrate the potential diagnostic use of these optical sensors able to discriminate between physiological and pathological conditions, substrates were tested against a $4 \mu \mathrm{M}$ aqueous solution of $A \beta$-42-peptide $(A \beta)$, which is one of the main pathological biomarkers for Alzheimer's disease found in the cerebrospinal fluid. Consistent with Rh6 G results, Raman spectra reported in figure 5 show that the intensity of characteristic peaks of $A \beta$ is higher in presence of metallic nanostructures which plasmonic band is in resonance with the excitation wavelength, while for bare PDMS the Raman profile is almost not visible. Therefore, metal nanostructures have a key role for detection of target analyte. To highlight the effective A $\beta$ Raman profile hidden by the peaks of bulky PDMS (figures 6(a) and (b)), A $\beta$ spectra were background subtracted and results are shown in figure 6(c) with bare PDMS, Cit-NSts and LA-NSts acquisition in order to validate SERS-enhancement effect. The complexity of protein Raman spectra contains a vast array of information and are mainly due to three types of contributes: the polypeptide backbones (amide bands) and 
the aromatic and non-aromatic amino acid side chain residues. The amide III band $\left(1230-1300 \mathrm{~cm}^{-1}\right)$ is slightly visible in the Raman spectra of $\mathrm{A} \beta$ solution fluxed through the bare PDMS microfluidic chamber. The intensity of the signal is enhanced in presence of metallic nanostructures, increasing as the overlap between plasmonic properties and excitation wavelength increases. The same trend, less noteworthy but still present, is recognized also for amide II band $\left(1520-1580 \mathrm{~cm}^{-1}\right)$, which is primarily related to C-N stretching, $\mathrm{C}-\mathrm{C}$ stretching and $\mathrm{N}-\mathrm{H}$ bending. This band is nearly undetectable in non-resonance Raman spectra, but thanks to the interaction of inelastic scattering field with resonant metallic nanostructured chamber, a weak peak occurs. It is also observed that amide I band $\left(1640-1680 \mathrm{~cm}^{-1}\right)$ is suppressed when SERS effect is present. The exact mechanism is not widely known in literature: it has been hypothesised that the absence of amide I band can be due to a parallel orientation of the peptide bonds to the metal surface; other groups proposed that the lack of Raman intensity could be ascribed to a poor specific adsorption of the peptide bonds to the metal surface, since no enhancement occurs in proximity of metal particle [44]. Resonances at $990 \mathrm{~cm}^{-1}$ and $1018 \mathrm{~cm}^{-1}$ are produced by the ring breathing and $\mathrm{C}-\mathrm{C}$ stretching of phenylalanine and tryptophan, while $1087 \mathrm{~cm}^{-1}$ peak is due to $\mathrm{C}-\mathrm{O}$ stretching; the 2-fold higher Raman intensity of these peaks generated by LA-NSts substrate confirms the best SERS performances of covalently stabilized NSts. Predominant peak in the $A \beta$ spectrum at $1430 \mathrm{~cm}^{-1}$, assigned as $\mathrm{C}-\mathrm{H}_{2}$ bending in amino acids with $\mathrm{C}=\mathrm{C}$ asymmetric stretching contributes, is 2- and 5-fold enhanced by Cit-NSts and LA-NSts PDMS substrates, respectively. Concentration is a crucial point since $\mathrm{A} \beta$ aggregation can occur when working at $\mu \mathrm{M}$ range. Working with very diluted samples could affect both Raman intensity and signal to noise ratio.

\section{Conclusions}

In the present work, we report the successful fabrication of novel SERS-microfluidic devices for synergic coupling with fiber-based Raman set-up and targeting of analytes diluted in liquid samples. To this end, optically transparent microfluidic devices were rapidly prototyped by combining PDMS replica molding with advanced 3D printing processes, thus guaranteeing high design flexibility as well as costs saving. Then, gold nanostars-devices were realized through selective immobilization of NPs whose optical and chemical properties were tailored to achieve higher reproducibility of the deposition process and SERS performances. NPs were engineered to control their plasmonic resonance as well as to strengthen their immobilization on the substrates avoiding or at least controlling aggregation phenomena. Finally, we see a great potential in further developing tunable SERS-fluidic devices by taking advantage of material chemistry and the synergetic coupling between optics and fluidics, as well as versatility in terms of analyte to be detected.

\section{Acknowledgments}

This work was supported by Ente Cassa di Risparmio di Firenze (private foundation). The authors also wish to acknowledge Dr. Olga Bibikova (Art Photonics, Berlin, Germany), Dr. Joao Lagarto and Dr. Enrico Baria (European Laboratory for Non-Linear Spectroscopy, Florence, Italy), Dr. Fulvio Ratto, Dr. Sonia Centi and Dr. Roberto Pini (Institute of Applied Physics 'N. Carrara', CNR-Florence, Italy) for their assistance in the experiments.

\section{ORCID iDs}

Caterina Credi (i) https://orcid.org/0000-0003-4565-5214

Elena Lenci (i) https://orcid.org/0000-0001-7408-2828

Andrea Trabocchi (1) https://orcid.org/0000-0003-1774-9301

\section{References}

[1] Lassmann H et al 1995 Cell death in Alzheimer's disease evaluated by DNA fragmentation in situ Acta Neuropathol. 89 35-41

[2] Wehmeyer K R, Halsall H B and Heineman W R 1985 Heterogeneous enzyme immunoasssay with electrochemical detection: competitive and 'sandwich'-type immunoassays Clin. Chem. 31 1546-9

[3] Mègraud F 1996 Advantages and disadvantages of current diagnostic tests for the detection of Helicobacter pylori Scand. J. Gastroenterol. Suppl. 31 57-62

[4] Eberhardt K, Stiebing C, Matthaüs C, Schmitt M and Popp J 2015 Advantages and limitations of Raman spectroscopy for molecular diagnostics: an update Expert Rev. Mol. Diagn. 15 773-87

[5] Fleischmann M, Hendra P J and McQuillan A J 1974 Raman spectra of pyridine adsorbed at a silver electrode Chem. Phys. Lett. 26 163-6

[6] Etchegoin P G and Le Ru E C 2010 Basic Electromagnetic Theory of SERS (New York: Wiley)

[7] Sharma B, Frontiera R R, Henry A-I, Ringe E and Van Duyne R P 2012 SERS: materials, applications, and the future Mater. Today 15 16-25 
[8] Jahn I J, Weber K, Bocklitz T W and Popp J 2017 Surface-enhanced Raman spectroscopy and micro fl uidic platforms: challenges, solutions and potential applications Analyst 142 1022-47

[9] Lim C, Hong J, Chung B G, DeMello A J and Choo J 2010 Optofluidic platforms based on surface-enhanced Raman scattering Analyst 135 837-44

[10] Wu L, Wang Z, Zong S and Cui Y 2014 Rapid and reproducible analysis of thiocyanate in real human serum and saliva using a droplet SERS-microfluidic chip Biosens. Bioelectron. 62 13-18

[11] Yılmaz B and Yılmaz F 2017 Lab-on-a-chip technology and its applications Omics Technologies and Bio-Engineering ed D Barh and V Azevedo (Amsterdam: Elsevier) p 145-53

[12] Nayak S, Blumenfeld N R, Laksanasopin T S and Samuel K 2017 Point-of-care diagnostics: recent developments in a connected age Anal. Chem. 89 102-23

[13] Ahn C H et al 2004 Disposable smart lab on a chip for point-of-care clinical diagnostics Proc. IEEE 92

[14] Fortuni B et al 2017 In situ synthesis of Au-shelled Ag nanoparticles on PDMS for flexible, long-life, and broad spectrum-sensitive SERS substrates Chem. Commun. 53 11298-301

[15] Strehle K R et al 2007 A reproducible surface-enhanced Raman spectroscopy approach. online SERS measurements in a segmented microfluidic system Anal. Chem. 79 1542-7

[16] Chang S, Combs Z A, Gupta M K, Davis R and Tsukruk V V 2010 In situ growth of silver nanoparticles in porous membranes for surface-enhanced Raman scattering ACS Appl. Mater. Interfaces 23333

[17] Yuan H, Fales A M, Khoury C G, Liu J and Vo-Dinh T 2013 Spectral characterization and intracellular detection of surface-enhanced Raman scattering (SERS)-encoded plasmonic gold nanostars J. Raman Spectrosc. 44 234-9

[18] Cicchi R et al 2014 Combined fluorescence-Raman spectroscopic setup for the diagnosis of melanocytic lesions J. Biophoton. 7 86-95

[19] Habermehl A et al 2017 Aerosol jet printing and roll-to-roll hot embossing Sensors 2017 1-11

[20] Credi C, Griffini G, Levi M and Turri S 2018 Biotinylated photopolymers for 3D-printed unibody lab-on-a-chip optical platforms Small 141702831

[21] Credi C et al 2017 Combining stereolithography and replica molding : on the way to superhydrophobic polymeric devices for photovoltaics Mater. Des. 133 143-53

[22] Id B K G, Id A R J, Id C J L and Goenner B L 2018 A review of current methods in microfluidic device fabrication and future commercialization prospects Inventions 201860

[23] Moolman M C, Huang Z, Krishnan S T and Kerssemakers J W J 2013 Electron beam fabrication of a microfluidic device for studying submicron-scale bacteria J. Nanobiotechnol. 1112

[24] Horváth B, Křivová B and Micro S H 2019 Nano engineering nanoimprint meets microfluidics : development of metal wires from nanoparticle ink filled capillaries Micro. Nano Eng. 3 22-30

[25] Ren B et al 2018 Facile fabrication of microfluidic surface-enhanced Raman scattering devices via lift-up lithography R. Soc. Open Sci. 5172034

[26] Hoelzle D, Lake M, Narciso C, Cowdrick K, Storey T, Zhang S, and Zartman J, 2015 Microfluidic device design, fabrication, and testing protocols Protocol Exchange 2015 1-26

[27] Kamei K, Mashimo Y, Koyama Y, Fockenberg C, Nakashima M, Li J and Chen Y 2015 3D printing of soft lithography mold for rapid production of polydimethylsiloxane-based microfluidic devices for cell stimulation with concentration gradients Biomed. Microdevices 1736

[28] Credi C et al 2019 Disposable and versatile optical sensors for SERS analysis of liquid samples by fiber-based spectroscopy Proc. SPIE $10872108720 Z$

[29] Mocan L et al 2016 Selective in vitro photothermal nano-therapy of MRSA infections mediated by IgG conjugated gold nanoparticles Sci. Rep. 639466

[30] Love J C, Estroff L A, Kriebel J K, Nuzzo R G and Whitesides G M 2005 Self-assembled monolayers of thiolates on metals as a form of nanotechnology Chem. Rev. 105 1103-70

[31] Guerrini L, Alvarez-Puebla R A and Pazos-Perez N 2018 Surface modifications of nanoparticles for stability in biological fluids Materials 11 1-28

[32] Tournebize J et al 2012 Role of gold nanoparticles capping density on stability and surface reactivity to design drug delivery platforms ACS Appl. Mater. Interfaces 4 5790-9

[33] Lin S-Y, Chen C, Chen -C-C, Tsai Y-T and Lin C-M 2004 Two-step functionalization of neutral and positively charged thiols onto citrate-stabilized Au nanoparticles J. Phys. Chem. B 108 2134-9

[34] Senthil Kumar P, Pastoriza-Santos I, Rodríguez-González B, De Abajo F J G and Liz-Marzán L M 2008 High-yield synthesis and optical response of gold nanostars Nanotechnology 19 1-7

[35] Shiohara A, Langer J, Polavarapu L and Liz-Marzán L M 2014 Solution processed polydimethylsiloxane/gold nanostar flexible substrates for plasmonic sensing Nanoscale 6 9817-23

[36] Il P Y, Im H, Weissleder R and Lee H 2015 Nanostar clustering improves the sensitivity of plasmonic assays Bioconjugate Chem. 26 1470-4

[37] Eddings M A, Johnson M A and Gale B K 2008 Determining the optimal PDMS-PDMS bonding technique for microfluidic devices J. Micromech. Microeng. 18067001

[38] Yuan H et al 2012 Gold nanostars: surfactant-free synthesis, 3D modelling, and two-photon photoluminescence imaging Nanotechnology 23075102

[39] Zhao J, Lui H, Mclean D I and Zeng H 2007 Automated autofluorescence background subtraction algorithm for biomedical raman spectroscopy Appl. Spectrosc. 61 1225-32

[40] Volkert A A, Subramaniam V, Ivanov M R, Goodman A M and Haes A J 2011 Salt-mediated self-assembly of thioctic acid on gold nanoparticles ACS Nano 5 4570-80

[41] Guo C and Yarger J L 2018 Characterizing gold nanoparticles by NMR spectroscopy Magn. Reson. Chem. 56 1074-82

[42] Niu W, Chua Y A A, Zhang W, Huang H and Lu X 2015 Highly symmetric gold nanostars: crystallographic control and surface-enhanced raman scattering property J. Am. Chem. Soc. 137 10460-3

[43] Mosier-Boss P A 2017 Review of SERS substrates for chemical sensing Nanomaterials 7142

[44] Kurouski D, Postiglione T, Deckert-Gaudig T, Deckert V and Lednev I K 2013 Amide I vibrational mode suppression in surface (SERS) and tip (TERS) enhanced Raman spectra of protein specimens Analyst 138 1665-73 